\title{
A Combined Segmentation and Registration Framework with a Nonlinear Elasticity Smoother
}

\author{
Carole Le Guyader ${ }^{1}$ and Luminita A. Vese $^{2}$ \\ ${ }^{1}$ IRMAR, UMR CNRS 6625 \\ Institut National des Sciences Appliquées de Rennes \\ 20, Avenue des Buttes de Coësmes, CS 14315, 35043 RENNES Cedex, France \\ carole.le-guyader@insa-rennes.fr \\ 2 Department of Mathematics, University of California, Los Angeles \\ Los Angeles, CA 90095-1555, USA \\ lvese@math.ucla.edu
}

\begin{abstract}
In this paper, we present a new non-parametric combined segmentation and registration method. The problem is cast as an optimization one, combining a matching criterion based on the active contour without edges 4 for segmentation, and a nonlinear-elasticity-based smoother on the displacement vector field. This modeling is twofold: first, registration is jointly performed with segmentation since guided by the segmentation process; it means that the algorithm produces both a smooth mapping between the two shapes and the segmentation of the object contained in the reference image. Secondly, the use of a nonlinearelasticity-type regularizer allows large deformations to occur, which makes the model comparable in this point with the viscous fluid registration method [7. Several applications are proposed to demonstrate the potential of this method to both segmentation of one single image and to registration between two images.
\end{abstract}

\section{Introduction}

Image registration and image segmentation are challenging issues that are encountered in a wide range of fields such as medical imaging (shape tracking, comparison of images taken at different instants, data fusion from images that have not necessarily been acquired with the same modality, comparison of data to a common reference frame), pattern recognition or geophysics, etc.

We propose in this paper a segmentation model based on the active contour model without edges [4], that is no longer solved in terms of level set functions. This is now solved using registration techniques. Therefore, a displacement field models the deformation of the initial curve into the final segmented boundary via registration. Thus, the binary segmentation functional [4,

$$
F\left(c_{1}, c_{2}, \phi\right)=\int_{\Omega}\left\{\nu_{1}\left|R-c_{1}\right|^{2} H(\phi)+\nu_{2}\left|R-c_{2}\right|^{2}(1-H(\phi))+\mu|\nabla H(\phi)|\right\} d \boldsymbol{x}
$$

( $R$ is the given image, $\phi$ is a level set function describing the unknown contour, $H$ is the Heaviside function), can be reformulated as a warping problem 
between the binary image defining the initial contour, and the (unknown) binary segmented image. Or the proposed model can also be used for registration between two images: having a segmentation of one of the images defined via a displacement field, this is used as initial guess in the "registration-segmentation" model, to segment/register the second image. The main ingredients of our proposed minimization model are thus the active contour model without edges 4, and registration via a non-linear elasticity smoother, solved in a particular simplified way. The unknown level set function $\phi$ is substituted by the unknown transformation, with an appropriate regularization as a substitute for the length term. Topology-preserving segmentation results can be obtained.

An extensive overview of registration techniques can be found in [24]. These can be partitioned into two classes: parametric and non-parametric. In the nonparametric methods (our framework) the problem is phrased as a functional minimization whose unknown is the displacement vector field $\boldsymbol{u}$. Denoting by $T$ the template, by $R$ the reference, the introduced functional combines a distance measure component $\mathcal{D}[R, T, \boldsymbol{u}]$ and a smoother on the displacement vector field $\mathcal{S}=$ $\mathcal{S}[\boldsymbol{u}]$ to remove the ill-posed character of the problem. Usually, the distance measure is intensity-driven and is chosen to be the $L^{2}$-norm of the difference between the deformed template and the reference (suitable when the images have been acquired through similar sensors), i.e. $\mathcal{D}[R, T, \boldsymbol{u}]=\frac{1}{2} \int_{\Omega}(T(\boldsymbol{x}+\boldsymbol{u})-R(\boldsymbol{x}))^{2} d \boldsymbol{x}$, but one could also use correlation-based or mutual information-based techniques 24.

Several methods to regularize the displacement vector field have been investigated. One is the elastic registration introduced by Broit [3, in which the objects to be registered are considered to be the observations of a same elastic body before and after being subjected to a deformation. The smoother $\mathcal{S}=\mathcal{S}[\boldsymbol{u}]$ is chosen to be the linearized elastic potential of the displacement vector field $\boldsymbol{u}$ and its expression integrates the Lamé coefficients $\lambda, \mu$ which reflect material properties. A drawback of this smoother is that it is not suitable for problems involving large deformations. To circumvent this problem, Christensen et al. 7 proposed a viscous fluid registration model in which objects are viewed as fluids evolving in accordance with the fluid-dynamic Navier-Stokes equations. However, this is a computationally expensive procedure.

In the diffusion registration model introduced by Fischer and Modersitzki [11, the smoother is based on the semi-norm of $H^{1}\left(\Omega, \mathbb{R}^{n}\right)$ of $\boldsymbol{u}=\left(u_{1}, \cdots, u_{n}\right)^{T}$, $\Omega$ being an open bounded subset of $\mathbb{R}^{n}$. Regularizing properties motivate this choice (it minimizes oscillations of all components of $\boldsymbol{u}$ ) rather than physical ones but here again only small deformations can be expected. In the "curvature"based registration model introduced by Fischer and Modersitzki [12], [13, the $\dot{H}^{2}$ (biharmonic) regularization is explored. Affine linear transformations belong to the kernel of the regularizer $\mathcal{S}[\boldsymbol{u}]$, which is not the case in elastic, viscous fluid or diffusion registration. But here again, transformations are restricted to small deformations. To circumvent this drawback, we propose in this paper a nonlinear elasticity-based smoother that allows larger deformations. 
Many improvements or alternatives of these non-parametric methods have been proposed. These include [14, [15, [37, [21, [20, [19]. By comparison with some of these methods, the only input required in our method is a fixed level set function representing the template image, that is, partitioning the image into two regions. Also, we jointly treat segmentation and registration: the distance measure is devised using the segmentation criterion [4, while registration is jointly performed, guided by the segmentation process. Our method applies to a particular class of images, since the binary criterion is being used. Before depicting our approach, we would like to mention previous work for joint segmentation and registration while stressing the main differences with our model.

In [38, Yezzi et al. also suggest to jointly treat segmentation and registration. The authors couple segmentation and registration as follows: denoting $R: \Omega \subset$ $\mathbb{R}^{2} \rightarrow \mathbb{R}$ and $T=\widehat{R}: \widehat{\Omega} \subset \mathbb{R}^{2} \rightarrow \mathbb{R}$ the two images containing a common object to be registered and segmented, find a closed curve $C \subset \Omega$ and a closed curve $\widehat{C} \subset \widehat{\Omega}$ related by $\widehat{C}=\mathbf{g}(C)$ where $\mathbf{g}: \mathbb{R}^{2} \rightarrow \mathbb{R}^{2}$ is an element of a finite dimensional group $G$ (for instance, the group of rigid motions) such that $C$ and $\widehat{C}$ correctly delineate the object contained respectively in $R$ and the one contained in $\widehat{R}$. Consequently, there are two unknowns, the closed curve $C \subset \Omega$ and the mapping $\mathbf{g}$. The authors exploit region-based active contour models [4] and minimize the energy:

$$
\begin{aligned}
& E(\mathbf{g}, C)=E_{1}(C)+E_{2}(\mathbf{g}(C)) \\
= & \int_{C_{\text {in }}}\left|R-c_{1}\right|^{2} d \boldsymbol{x}+\int_{C_{\text {out }}}\left|R-c_{2}\right|^{2} d \boldsymbol{x}+\int_{\widehat{C}_{\text {in }}}\left|\widehat{R}-\widehat{c_{1}}\right|^{2} d \boldsymbol{x}+\int_{\widehat{C}_{\text {out }}}\left|\widehat{R}-\widehat{c_{2}}\right|^{2} d \boldsymbol{x}
\end{aligned}
$$

with $C_{\text {in }}$ and $C_{\text {out }}$ the regions inside and outside $C, c_{1}$ and $c_{2}$ the mean values of $R$ on $C_{\text {in }}$ and $C_{\text {out }}$, and with $\widehat{C}_{\text {in }}$ and $\widehat{C}_{\text {out }}$ the regions inside and outside $\widehat{C}, \widehat{c_{1}}$ and $\widehat{c_{2}}$ the mean values of $\widehat{R}$ on $\widehat{C}_{\text {in }}$ and $\widehat{C}_{\text {out }}$. The main differences with our model are: the contours $C$ and $\widehat{C}$ are jointly deformed here through a combination of segmentation and registration methods while in our model, we assume that the object in the template image has already been detected (we could have considered a problem with two unknowns as well). It means that the energy-minimization problem is only written in terms of the unknown contour $\widehat{C}$. Segmentation is performed using a registration approach as in 38. The model is cast in the level set setting, which allows a straightforward modeling of the evolving curve. Contrary to [38, the class of admissible deformations (rigid, etc...) is not an input in our model. Their model, first exposed in the context of rigid deformations, has then been extended to non-rigid motions [35], [34, [29].

We would also like to mention the interesting work by Lord et al. 222] which uses a matching criterion based on metric structure comparison. The authors propose a unified method that simultaneously treats segmentation and registration by introducing two unknowns in the process: the deformation map and the segmenting curve. The segmentation process is guided by the registration map. The matching criterion, unlike classical registration methods, rests on the minimization of deviation from isometry. The matching criterion introduced is based on the metric structure comparison of the surfaces, more precisely on their first 
fundamental form, and on a homogeneity constraint as in [4. Thus contrary to our model in which the expected curve (implicitly represented as the zero level set of a Lipschitz function) delineates two regions with homogeneous intensity, their criterion is still based on metric structure comparisons to disconnect normal regions from abnormal ones.

We would also like to mention the related work by Vemuri et al. 31, 32. The authors propose a coupled PDE model to perform both segmentation and registration. In the first PDE, the level sets of the source image are evolved along their normals with a speed defined as the difference between the target and the evolving source image. The second PDE allows to explicitly retrieve the displacement vector field. In particular, in the work of Vemuri-Chen 30 for joint registration and segmentation, the piecewise-smooth level set segmentation model from 33 is combined with prior shape information through global alignment. As will be seen below, our model is different from the one in [30]. We also refer the reader to [5] in which a geodesic-active-contour-based model including a shape prior is presented and [6] in which a shape prior is incorporated this time in the Mumford-Shah model.

Related work is presented in [10, on an atlas-based segmentation of medical images locally constrained by level sets. We wish to refer to a segmentation method, different from ours, that also uses nonlinear elasticity to define the deformation of the evolving contour or surface in Rouchdy et al. [27]. The segmentation criterion is based on the gradient vector flow [36], and a deformation field is computed via non-linear elasticity using the finite element method. For completeness, we also refer the reader to [2], 23] for a variational registration method for large deformations, to [26, for a much related work which also uses nonlinear elasticity regularization but which is implemented using the finite element method, and to [9], a related work that uses nonlinear elasticity principles but different from our proposed approach. More details of the proposed method are presented in [18].

\section{Description of the Proposed Model}

As mentioned in Sect. 1, the scope of the proposed method is twofold:

- devise a model in which segmentation and registration are jointly performed.

- large and smooth deformations must be authorized, while keeping the deformation map topology-preserving.

We see in the sequel how these criteria are fulfilled.

Distance Measure Criterion. Let $\Omega$ be a bounded open subset of $\mathbb{R}^{n}$. For the purpose of illustration, we consider the case $n=2$. Let us denote by $R: \bar{\Omega} \rightarrow \mathbb{R}$ the "reference" image to be segmented (later we will discuss how the proposed method can be used for registration between a template image $T: \bar{\Omega} \rightarrow \mathbb{R}$ and the reference image $R$; initially, our method is defined as a segmentation method based on [4). Let $\Phi_{0}$ be a given Lipschitz level set function. Denoting by $\mathcal{C}$ the zero level set of $\Phi_{0}$ and $w \subset \Omega$ the open set it delineates, $\Phi_{0}$ is such that: 
$\mathcal{C}=\left\{\boldsymbol{x} \in \Omega \mid \Phi_{0}(\boldsymbol{x})=0\right\}, w=\left\{\boldsymbol{x} \in \Omega \mid \Phi_{0}(\boldsymbol{x})>0\right\}, \Omega \backslash \bar{w}=\left\{\boldsymbol{x} \in \Omega \mid \Phi_{0}(\boldsymbol{x})<0\right\}$

The deformation of the evolving curve is made in order to satisfy a segmentation criterion. Indeed, the distance measure we introduce is related to the fitting term of the active contours without edges model [4. In this way, registration and segmentation are correlated and we expect, at the end of the process, to obtain the segmentation of the reference image as well as a smooth deformation map. It results in a region-based intensity approach and no longer in a pointwise process as usually done. The idea is to find a smooth displacement vector field $\boldsymbol{u}=\left(u_{1}, u_{2}\right): \Omega \rightarrow \mathbb{R}^{2}, \boldsymbol{x} \mapsto\left(u_{1}(\boldsymbol{x}), u_{2}(\boldsymbol{x})\right) \in \Omega$, for each $\boldsymbol{x} \in \Omega$, such that the zero level line of $\Phi$ defined by $\Phi(\boldsymbol{x})=\Phi_{0}(\boldsymbol{x}+\boldsymbol{u}(\boldsymbol{x}))$ fits the boundary of the object to be warped in the given "reference" image. Denoting by $H$ the onedimensional Heaviside function, by $\nu_{1}, \nu_{2}>0$ two fixed parameters and $c_{1}$ and $c_{2}$ being two unknown constants depending on $\Phi_{0}, R$ and $\boldsymbol{u}$, the distance measure functional $F_{d}$ (the segmentation criterion) is defined by:

$$
\begin{aligned}
F_{d}\left(c_{1}, c_{2}, \boldsymbol{u}\right) & =\nu_{1} \int_{\Omega}\left|R(\boldsymbol{x})-c_{1}\right|^{2} H\left(\Phi_{0}(\boldsymbol{x}+\boldsymbol{u}(\boldsymbol{x}))\right) d \boldsymbol{x} \\
& +\nu_{2} \int_{\Omega}\left|R(\boldsymbol{x})-c_{2}\right|^{2}\left(1-H\left(\Phi_{0}(\boldsymbol{x}+\boldsymbol{u}(\boldsymbol{x}))\right)\right) d \boldsymbol{x} .
\end{aligned}
$$

We need to add a regularization term of the form $F_{\text {reg }}(\boldsymbol{u})$ to (1), which is a substitute for the length term of the evolving curve in [4, and therefore the unknown $\Phi(\boldsymbol{x})$ from [4] is substituted by $\Phi_{0}(\boldsymbol{x}+\boldsymbol{u}(\boldsymbol{x}))$, with $\Phi_{0}$ fixed now. Thus, we obtain a binary segmentation method that can also be used for registration.

Introduction of a Nonlinear Elasticity-Based Regularizer. A regularizing term $F_{r e g}$ is now introduced to ensure the smoothness of the displacement vector field $\boldsymbol{u}$. To allow large displacements, we introduce a nonlinear-elasticitybased smoother. We propose to view the deformation of the initial contour into the final segmented contour as the deformation undergone by St. Venant-Kirchhoff materials. These materials are homogeneous, isotropic, hyperelastic and the axiom of frame indifference is satisfied (see [8] for further details). Let us denote by $\varepsilon$ the Green-St. Venant strain tensor defined by: $\varepsilon=\frac{1}{2}(\boldsymbol{C}-\boldsymbol{I})$ with $\boldsymbol{C}=\nabla \boldsymbol{\varphi}^{T} \nabla \boldsymbol{\varphi}$, $\boldsymbol{\varphi}$ being the deformation such that $\boldsymbol{\varphi}=\boldsymbol{I} \boldsymbol{d}+\boldsymbol{u}, \nabla \boldsymbol{\varphi}$ being the Jacobian matrix and $I$ denoting the identity matrix. We have equivalently $\boldsymbol{\varepsilon}=\boldsymbol{\varepsilon}(\boldsymbol{u})=\frac{1}{2}\left(\nabla \boldsymbol{u}^{T}+\nabla \boldsymbol{u}+\right.$ $\left.\nabla \boldsymbol{u}^{T} \nabla \boldsymbol{u}\right)$. The strain tensor is a measure of the deviation between a given deformation and a rigid deformation for which $\boldsymbol{C}=\boldsymbol{I}$. As stressed by Ciarlet ( [8]), St. Venant-Kirchhoff materials are the simplest ones among nonlinear models (large strains are also possible when the stress is small, however a linear relation implies that the stress is small if and only if the strain is small). The stored energy of St. Venant-Kirchhoff materials [8] is given by $W(\varepsilon)=\frac{\lambda}{2}(\operatorname{tr} \varepsilon)^{2}+\mu \operatorname{tr} \varepsilon^{2}$. Thus, the nonlinear elasticity regularizer that will be coupled with the distance measure functional $F_{d}$ is defined by:

$$
F_{\text {reg }}(\boldsymbol{u})=\int_{\Omega} W(\varepsilon(\boldsymbol{u})) d \boldsymbol{x}=\int_{\Omega}\left\{\frac{\lambda}{2}(\operatorname{tr} \varepsilon(\boldsymbol{u}))^{2}+\mu \operatorname{tr} \varepsilon^{2}(\boldsymbol{u})\right\} d \boldsymbol{x} .
$$


Although this functional does not satisfy known theoretical assumptions (the stored energy function is not polyconvex; it is also not rank-1 convex and consequently not quasiconvex, which raises a drawback of theoretical nature since the introduced functional is not lower semi-continuous on $W^{1,4}$ ) to insure existence of minimizers, we can expect to get, in practice, better results than those obtained with linearized models, as will be demonstrated next. The computation of the Euler-Lagrange equation satisfied by $\boldsymbol{u}$ is cumbersome. Following the idea of the more theoretical work [25], we propose to circumvent this issue by introducing a second unknown, a matrix auxiliary variable $\boldsymbol{V}$, which approximates the Jacobian matrix of $\boldsymbol{u}$. The nonlinear elasticity regularizer is thus applied to $\boldsymbol{V}$ and no longer to $\nabla \boldsymbol{u}$, that is, the nonlinearity is no longer in the derivatives of the unknown $\boldsymbol{u}$. Also, as the matrix variable $\boldsymbol{V}$ is introduced to mimic the Jacobian matrix of $\boldsymbol{u}$, an additional term based on the Frobenius norm denoted by $\|\cdot\|_{F}$ of $\nabla \boldsymbol{u}-\boldsymbol{V}$ is incorporated in the modeling. More precisely, letting $\widehat{\boldsymbol{V}}=\frac{\boldsymbol{V}^{\boldsymbol{T}}+\boldsymbol{V}+\boldsymbol{V}^{\boldsymbol{T}} \boldsymbol{V}}{2}$ and $\alpha>0$ a tuning parameter, we redefine the smoothing functional $\stackrel{2}{F}_{r e g}=F_{r e g}(\boldsymbol{u}, \boldsymbol{V})$ by:

$$
F_{r e g}(\boldsymbol{u}, \boldsymbol{V})=\int_{\Omega} W(\widehat{\boldsymbol{V}}) d \boldsymbol{x}+\frac{\alpha}{2} \int_{\Omega}\|\nabla \boldsymbol{u}-\boldsymbol{V}\|_{F}^{2} d \boldsymbol{x} .
$$

In the limit, as $\alpha \rightarrow+\infty$, we obtain $\nabla \boldsymbol{u} \simeq V$ in the $L^{2}$-topology.

Total Energy Functional. The total energy $E_{\text {total }}$ considered in the remainder of this work is given by:

$$
E_{\text {total }}\left(c_{1}, c_{2}, \boldsymbol{u}, \boldsymbol{V}\right)=F_{d}\left(c_{1}, c_{2}, \boldsymbol{u}\right)+F_{\text {reg }}(\boldsymbol{u}, \boldsymbol{V}) .
$$

Evolution Problem. We give the form of the associated Euler-Lagrange equations in the two-dimensional case. In the calculations, the Heaviside function is replaced by a smooth version denoted by $H_{\epsilon}$ and $H_{\epsilon}^{\prime}=\delta_{\epsilon}$, regularization of the Dirac measure. Fixing $\boldsymbol{u}$ and $\boldsymbol{V}$ and minimizing $E_{\text {total }}\left(c_{1}, c_{2}, \boldsymbol{u}, \boldsymbol{V}\right)$ with respect to $c_{1}$ and $c_{2}$ yields, as in [4]:

$$
c_{1}=\frac{\int_{\Omega} R(\boldsymbol{x}) H\left(\Phi_{0}(\boldsymbol{x}+\boldsymbol{u}(\boldsymbol{x}))\right) d \boldsymbol{x}}{\int_{\Omega} H\left(\Phi_{0}(\boldsymbol{x}+\boldsymbol{u}(\boldsymbol{x}))\right) d \boldsymbol{x}}, \quad c_{2}=\frac{\int_{\Omega} R(\boldsymbol{x})\left(1-H\left(\Phi_{0}(\boldsymbol{x}+\boldsymbol{u}(\boldsymbol{x}))\right)\right) d \boldsymbol{x}}{\int_{\Omega}\left(1-H\left(\Phi_{0}(\boldsymbol{x}+\boldsymbol{u}(\boldsymbol{x}))\right)\right) d \boldsymbol{x}} .
$$

Computing the first variation of functional $F_{d}\left(c_{1}, c_{2}, \boldsymbol{u}\right)$ in (1) with respect to $\boldsymbol{u}$ gives the following gradient:

$$
\partial_{\boldsymbol{u}} F_{d}\left(c_{1}, c_{2}, \boldsymbol{u}\right)=\left(\nu_{1}\left(R-c_{1}\right)^{2}-\nu_{2}\left(R-c_{2}\right)^{2}\right) \delta_{\epsilon}\left(\Phi_{0}(\boldsymbol{x}+\boldsymbol{u}(\boldsymbol{x}))\right) \nabla \Phi_{0}(\boldsymbol{x}+\boldsymbol{u}(\boldsymbol{x})) .
$$

Also, computing the first variation of functional $F_{\text {reg }}(\boldsymbol{u}, \boldsymbol{V})$ in (3) with respect to $\boldsymbol{u}$ gives only linear differential equations in each $u_{i}$ :

$$
\partial_{u_{k}} F_{r e g}(\boldsymbol{u}, \boldsymbol{V})=-\alpha\left(\triangle u_{k}-\left(\frac{\partial v_{k 1}}{\partial x_{1}}+\frac{\partial v_{k 2}}{\partial x_{2}}\right)\right), k=1,2 .
$$


To finish, setting $\boldsymbol{V}=\left(v_{i j}\right)_{1 \leq i, j \leq 2}$ and letting

$$
\begin{gathered}
c_{01}=v_{11}+v_{22}+\frac{1}{2}\left(v_{11}^{2}+v_{12}^{2}+v_{21}^{2}+v_{22}^{2}\right), c_{02}=2 v_{11}+v_{11}^{2}+v_{21}^{2} \\
c_{03}=2 v_{22}+v_{12}^{2}+v_{22}^{2}, \quad c_{04}=v_{12}+v_{21}+v_{11} v_{12}+v_{21} v_{22},
\end{gathered}
$$

we obtain:

$$
\begin{aligned}
& \partial_{v_{11}} F_{r e g}(\boldsymbol{u}, \boldsymbol{V})=\alpha\left(v_{11}-\frac{\partial u_{1}}{\partial x_{1}}\right)+\left(\lambda c_{01}+\mu c_{02}\right)\left(1+v_{11}\right)+\mu c_{04} v_{12} . \\
& \partial_{v_{12}} F_{r e g}(\boldsymbol{u}, \boldsymbol{V})=\alpha\left(v_{12}-\frac{\partial u_{1}}{\partial x_{2}}\right)+\left(\lambda c_{01}+\mu c_{03}\right) v_{12}+\mu c_{04}\left(1+v_{11}\right) . \\
& \partial_{v_{21}} F_{r e g}(\boldsymbol{u}, \boldsymbol{V})=\alpha\left(v_{21}-\frac{\partial u_{2}}{\partial x_{1}}\right)+\left(\lambda c_{01}+\mu c_{02}\right) v_{21}+\mu c_{04}\left(1+v_{22}\right) . \\
& \partial_{v_{22}} F_{r e g}(\boldsymbol{u}, \boldsymbol{V})=\alpha\left(v_{22}-\frac{\partial u_{2}}{\partial x_{2}}\right)+\left(\lambda c_{01}+\mu c_{03}\right)\left(1+v_{22}\right)+\mu c_{04} v_{21} .
\end{aligned}
$$

We solve the Euler-Lagrange equations in $\boldsymbol{u}$ and $\boldsymbol{V}$ using gradient descent, parameterizing the descent direction by an artificial time $t \geq 0$. Systems of 4 and 2 equations are obtained (solved by semi-implicit finite difference schemes),

$$
\frac{\partial \boldsymbol{V}}{\partial t}=-\partial_{V} F_{r e g}(\boldsymbol{u}, \boldsymbol{V}), \quad \frac{\partial \boldsymbol{u}}{\partial t}=-\partial_{\boldsymbol{u}} F_{d}\left(c_{1}, c_{2}, \boldsymbol{u}\right)-\partial_{\boldsymbol{u}} F_{r e g}(\boldsymbol{u}, \boldsymbol{V}),
$$

equipped with the boundary conditions $\boldsymbol{u}=\mathbf{0}_{\mathbb{R}^{2}}$ on $\partial \Omega$ and with the initial conditions $\boldsymbol{u}(x, 0)=\mathbf{0}_{\mathbb{R}^{2}}$ and $\boldsymbol{V}=\mathbf{0}_{M_{2}(\mathbb{R})}$.

In most cases, no regridding is necessary. Nevertheless, in the algorithm, we have used a regridding technique quite similar to the one proposed by Christensen et al. [7. The Jacobian $\operatorname{det}(\nabla(\boldsymbol{I} \boldsymbol{d}+\boldsymbol{u}))$ is monitored and if it drops below a defined threshold in some parts of the image, the process is reinitialized. The only change is that instead of doing the reinitialization step with the last deformed template as done in [7, we use the last deformed level set function $\Phi_{0}(\cdot+\boldsymbol{u}(\cdot))$. The overall displacement $\boldsymbol{u}$ is reconstructed similarly to [7].

\section{$3 \quad$ Numerical Experiments}

We conclude the paper by presenting several results on both synthetic and real images in 2 dimensions. In most experiments, $\nu_{1}=\nu_{2}=1$ but when dealing with complex topologies involving long and thin concavities, these parameters have been increased up to 2.5. The $\mathcal{C}^{\infty}$ regularization of the Heaviside function [4] is $H_{\epsilon}(z)=\frac{1}{2}\left(1+\frac{2}{\pi} \arctan \frac{z}{\epsilon}\right)$.

Our first experimental test in Fig. 1 is an academic one and is similar to those performed by Modersitzki in 24] (we refer to pages 114-115, 129-130, 150-153, 168-170 for comparisons using linear elasticity, diffusion, curvature, or the viscous fluid method), with the goal to illustrate that the model easily handles large displacements while segmenting the reference object. The problem is to warp a 

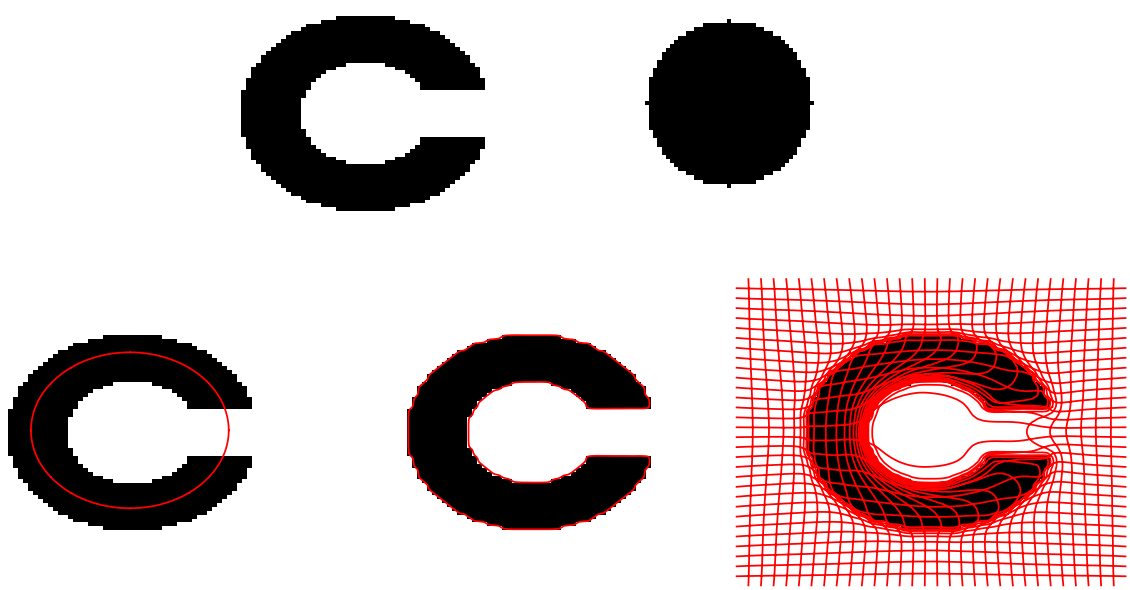

Fig. 1. Top: left, the reference image; right the template. Bottom: left, the boundary of the disk (zero level set of $\Phi_{0}$ ) superimposed on the reference image; middle, the segmentation of the letter C; right, deformed grid using nonlinear elasticity regularization.
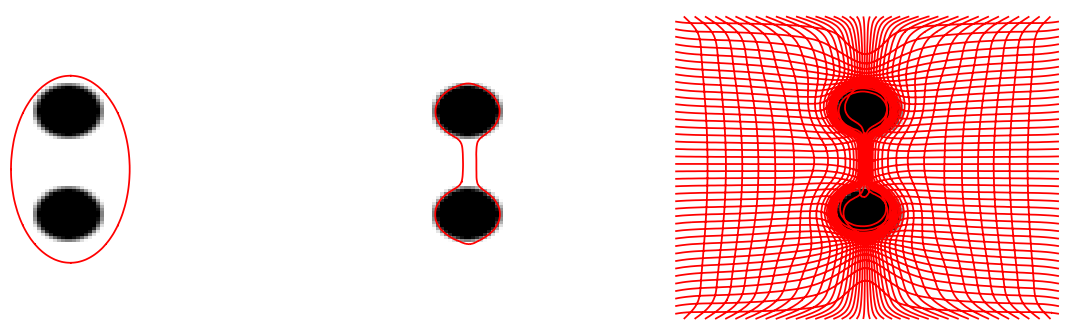

Fig. 2. Left, boundary of the ellipse (zero level set of $\Phi_{0}$ ) superimposed on the reference image; middle, the topology-preserving segmentation of the two disks; right, deformed grid using nonlinear elasticity regularization

black disk to the letter $\mathrm{C}$ both defined on the same image domain. The given data are the template and reference images as well as the curve delineating the disk boundary. We wish to demonstrate that our method qualitatively performs in a way similar to the fluid model without requiring the expensive Navier-Stokes solver employed for its numerical discretization, and provides two results: the segmentation of the reference image as well as a smooth displacement vector field $\boldsymbol{u}$. The implementation is simple, based on finite difference schemes, and allows to remove the nonlinearity in the derivatives of the unknown $\boldsymbol{u}$. The method allows large deformations unlike the linear elasticity model, diffusion model, curvature-based model for which the registration cannot be accomplished, the images differing too much (see pages 114-115, 150-153, 168-171 from [24]). In this example, three regridding steps were necessary: the transformation was considered as admissible if the Jacobian exceeded 0.01. Note that regridding steps were also necessary with the fluid registration model. 

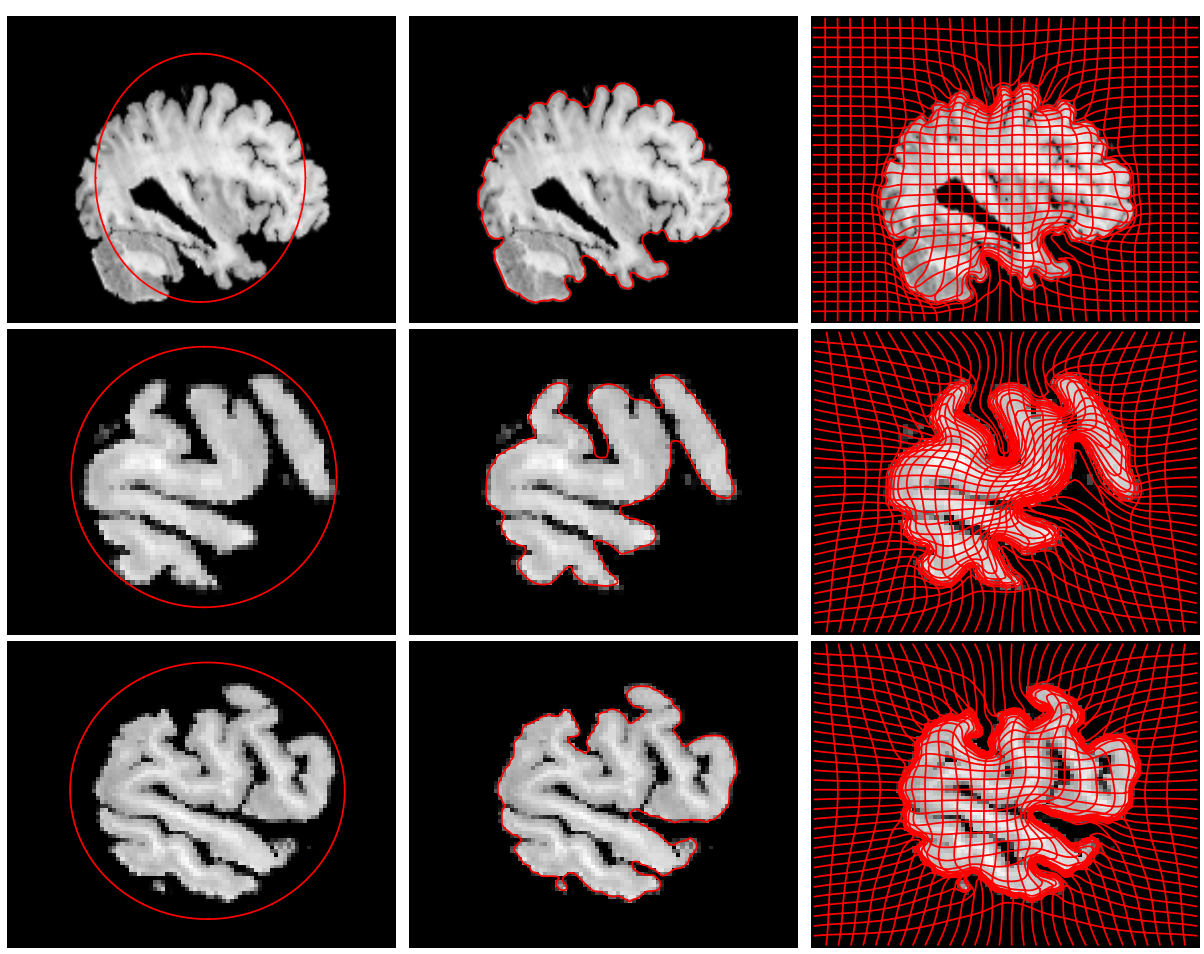

Fig. 3. Topology-preserving segmentation of three complex slices of the brain. Left, the boundary of the disk (zero level set of $\Phi_{0}$ ) superimposed on the reference image; middle, the segmentation of the slice of the brain; right, deformed grid using nonlinear elasticity regularization.

The second example in Fig. 2 illustrates how the method can be used in the case of topology-preserving segmentation ([16], [1], [28, [17] on this topic). This synthetic reference image represents two disks (similar to tests performed in prior related works [16], [28], [17]). The template image, defined on the same image domain is made of a black ellipse such that, when superimposed on the reference image, its boundary encloses the two disks. We aim at segmenting these two disks while maintaining the same topology throughout the process (one pathconnected component) and at obtaining a smooth displacement vector field $\boldsymbol{u}$. In this example, two regridding steps were necessary: the transformation was considered as admissible if the Jacobian exceeded 0.01.

The method has been tested on complex slices of brain data. The goal is to register a disk to the outer boundary of the brain with topology preservation. In Fig. 3, the template image, defined on the same image domain, is made of a disk (shown superimposed on the reference). Two regridding steps were necessary for the first row, and 3-4 regridding steps for the 2nd and 3rd rows: the transformation was considered as admissible if the Jacobian exceeded 0.01 . 


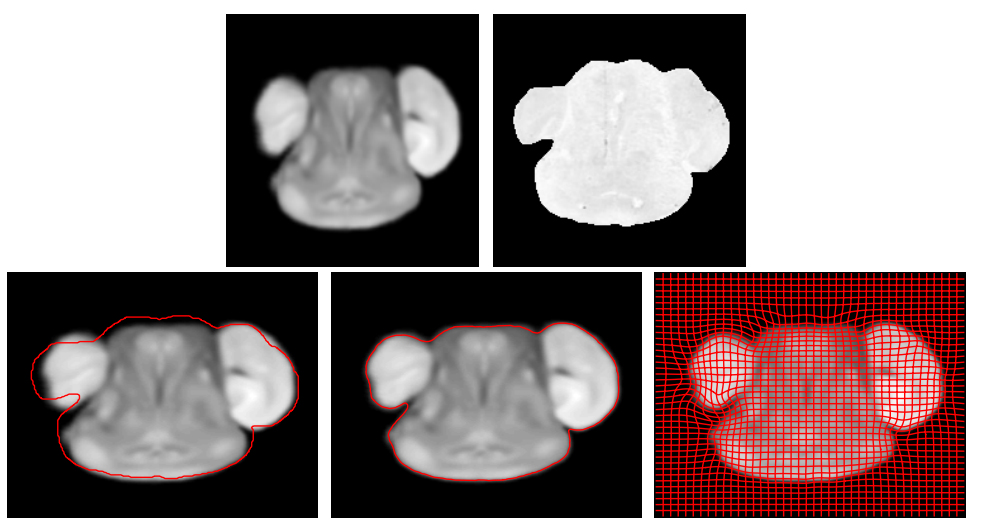

Fig. 4. Top: left, reference $R$; right, template $T$ (mouse atlas and gene data). Bottom, left to right: contour obtained by the proposed algorithm segmenting template $T$ (starting with $\Phi_{0}$ defining a disk), superimposed over the reference $R$; segmented reference, using as $\Phi_{0}$ the output contour detected at the previous step; final deformed grid using nonlinear elasticity smoother.

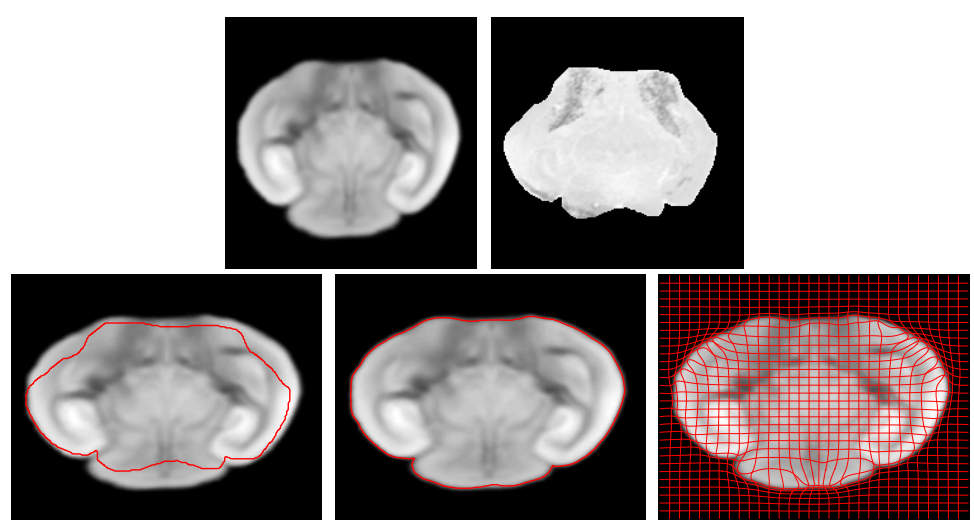

Fig. 5. Experiment exactly as in Fig. 4

Another medical application, as shown in Fig. 4 and Fig. 5 is proposed for mapping mouse gene data to an atlas. First, the proposed method is applied to the gene data, using $\Phi_{0}$ defining a disk, to segment it and extract a contour; then the method is applied again using as $\Phi_{0}$ the new contour, to segment the atlas data. In the process, we obtain a smooth deformation between the gene and the atlas data. No regridding step was necessary for Fig. 4

\section{Acknowledgments}

This work was supported in part by the National Institutes of Health (NIH) through the NIH Roadmap for Medical Research Grant U54 RR021813 entitled 
Center for Computational Biology, and by the National Science Foundation Grant DMS 0312222.

\section{References}

1. Alexandrov, O., Santosa, F.: A topology-preserving level set method for shape optimization. J. Comput. Phys. 204(1), 121-130 (2005)

2. Beg, F., Miller, M., Trouvé, A., Younes, L.: Computing large deformation metric mappings via geodesic flows of diffeomorphisms. IJCV 61(2), 139-157 (2005)

3. Broit, C.: Optimal Registration of Deformed Images. PhD thesis, Computer and Information Science, University of Pensylvania (1981)

4. Chan, T., Vese, L.: Active Contours Without Edges. IEEE Trans. Image Process. 10(2), 266-277 (2001)

5. Chen, Y., Thiruvenkadam, H., Tagare, H., Huang, F., Wilson, D.: On the Incorporation of Shape Priors in Geometric Active Contours. In: IEEE Workshop on VLSM, pp. 145-152 (2001)

6. Chen, Y., Thiruvenkadam, H., Gopinath, K., Brigg, R.: Image Registration Using the Mumford-Shah Functional and Shape Information. In: World Multiconference on Systems, Cybernetics and Informatics, pp. 580-583 (2002)

7. Christensen, G.E., Rabbitt, R.D., Miller, M.I.: Deformable Templates Using Large Deformation Kinematics. IEEE Trans. Image Process. 5(10), 1435-1447 (1996)

8. Ciarlet, P.G.: Elasticité Tridimensionnelle. Masson (1985)

9. Droske, M., Rumpf, M.: A variational approach to non-rigid morphological registration. SIAM J. Appl. Math. 64(2), 668-687 (2004)

10. Duay, V., Houhou, N., Thiran, J.-P.: Atlas-based segmentation of medical images locally constrained by level sets. In: ICIP, vol. 2 (2005)

11. Fischer, B., Modersitzki, J.: Fast Diffusion Registration. AMS Contemporary Mathematics. Inverse Problems, Image Analysis, and Medical Imaging 313, 117129 (2002)

12. Fischer, B., Modersitzki, J.: Curvature based image registration. JMIV 18(1), 81$85(2003)$

13. Fischer, B., Modersitzki, J.: A Unified Approach to Fast Image Registration and a New Curvature Based Registration Technique. Linear Algebra and its applications 380, 107-124 (2004)

14. Haber, E., Modersitzki, J.: Numerical methods for volume preserving image registration. Inverse problems 20(5), 1621-1638 (2004)

15. Haber, E., Modersitzki, J.: Image Registration with Guaranteed Displacement Regularity. Int. J. Comput. Vision 71(3), 361-372 (2007)

16. Han, X., Xu, C., Prince, J.L.: A Topology Preserving Level Set Method for Geometric Deformable Models. IEEE Trans. Pattern Anal. Mach. Intell. 25(6), 755-768 (2003)

17. Le Guyader, C., Vese, L.: Self-repelling snakes for topology-preserving segmentation models. IEEE Trans. Image Process. 17(5), 767-779 (2008)

18. Le Guyader, C., Vese, L.: A Combined Segmentation and Registration Framework with a nonlinear Elasticity Smoother. UCLA C.A.M. Report 08-16 (2008)

19. Leow, A., Chiang, M.-C., Protas, H., Thompson, P., Vese, L., Huang, H.S.C.: Linear and Non-Linear Geometric Object Matching with Implicit Representation. In: Proc. 17th ICPR, vol. 3, pp. 710-713 (2004) 
20. Liao, W.-H., Khuu, A., Bergsneider, M., Vese, L., Huang, S.-C., Osher, S.: From Landmark Matching to Shape and Open Curve Matching: A Level Set Approach. UCLA CAM Report 02-59 (2002)

21. Liao, W.-H., Yu, C.-L., Bergsneider, M., Vese, L., Huang, S.-C.: A New Framework of Quantifying Differences Between Images by Matching Gradient Fields and Its Application to Image Blending. In: Nuclear Science Symposium Conference Record, vol. 2, pp. 1092-1096. IEEE, Los Alamitos (2002)

22. Lord, N.A., Ho, J., Vemuri, B.C., Eisenschenk, S.: Simultaneous Registration and Parcellation of Bilateral Hippocampal Surface Pairs for Local Asymmetry Quantification. IEEE Trans. Med. Imaging 26(4), 417-478 (2007)

23. Miller, M., Trouvé, A., Younes, L.: On the metrics and Euler-Lagrange equations of computational anatomy. Annu. Rev. B. Eng. 4, 375-405 (2002)

24. Modersitzki, J.: Numerical Methods for Image Registration. Oxford University Press, Oxford (2004)

25. Negrón Marrero, P.V.: A numerical method for detecting singular minimizers of multidimensional problems in nonlinear elasticity. Numerische Mathematik 58, 135-144 (1990)

26. Rabbitt, R.D., Weiss, J.A., Christensen, G.E., Miller, M.I.: Mapping of hyperelastic deformable templates using the finite element method. In: Proceedings SPIE, vol. 2573, pp. 252-265 (1995)

27. Rouchdy, Y., Pousin, J., Schaerer, J., Clarysse, P.: A nonlinear elastic deformable template for soft structure segmentation: application to the heart segmentation in MRI. IP 23, 1017-1035 (2007)

28. Sundaramoorthi, G., Yezzi, A.: Global regularizing flows with topology preservation for active contours and polygons. IEEE Trans. Image Process. 16(3), 803-812 (2007)

29. Unal, G.B., Slabaugh, G.G.: Coupled PDE's for non-rigid registration and segmentation. In: CVPR, pp. 168-175 (2004)

30. Vemuri, B., Chen, Y.: Joint image registration and segmentation. In: Osher, S., Paragios, N. (eds.) Geometric Level Set Methods, pp. 251-269 (2003)

31. Vemuri, B., Ye, J., Chen, Y., Leonard, C.: A level-set based approach to image registration. In: IEEE Workshop on Mathematical Methods in Biomedical Image Analysis, pp. 86-93 (2000)

32. Vemuri, B., Ye, J., Chen, Y., Leonard, C.: Image Registration via level-set motion: Applications to atlas-based segmentation. Medical Image Analysis 7(1), 1-20 (2003)

33. Vese, L., Chan, T.: A Multiphase Level Set Framework for Image Segmentation Using the Mumford and Shah Model. IJCV 50(3), 271-293 (2002)

34. Wang, F., Vemuri, B.C.: Simultaneous registration and segmentation of anatomical structures from brain MRI. In: Duncan, J.S., Gerig, G. (eds.) MICCAI 2005. LNCS, vol. 3749, pp. 17-25. Springer, Heidelberg (2005)

35. Xiaohua, C., Brady, J.M., Rueckert, D.: Simultaneous segmentation and registration of medical images. In: Barillot, C., Haynor, D.R., Hellier, P. (eds.) MICCAI 2004. LNCS, vol. 3216, pp. 663-670. Springer, Heidelberg (2004)

36. Xu, C., Prince, J.L.: Snakes, shapes, and gradient vector flow. IEEE Trans. Image Process. 7, 359-369 (1998)

37. Yanovsky, I., Thompson, P.M., Osher, S., Leow, A.D.: Topology Preserving LogUnbiased Nonlinear Image Registration: Theory and Implementation. In: IEEE Conf. on CVPR (2007)

38. Yezzi, A., Zollei, L., Kapur, T.: A variational framework for joint segmentation and registration. IEEE-MMBIA, 44-51 (2001) 